Revista Arbitrada Interdisciplinaria KOINONIA

Año 2020. Vol V. №2. Especial: Saber y Tecnología Popular Hecho el depósito de Ley: FA2016000010

ISSN: 2542-3088

FUNDACIÓN KOINONIA (F.K). Santa Ana de Coro. Venezuela.

José Enrique Guevara-Valle; Andrea Raquel Zúñiga-Paredes; Edmundo José Jalón-Arias

http://dx.doi.org/10.35381/r.k.v5i2.925

\title{
Aplicativo web para la creación y toma de evaluaciones a estudiantes del nivel secundario
}

\section{Web application for the creation and taking of evaluations to high school students}

\author{
José Enrique Guevara-Valle \\ jegv-jose@hotmail.com \\ Universidad Regional Autónoma de los Andes, Quevedo \\ Ecuador \\ https://orcid.org/0000-0002-8386-5338 \\ Andrea Raquel Zúñiga-Paredes \\ uq.andreazuniga@uniandes.edu.ec \\ Universidad Regional Autónoma de los Andes, Quevedo \\ Ecuador \\ https://orcid.org/0000-0002-6231-262X \\ Edmundo José Jalón-Arias \\ edmundojalon@uniandes.edu.ec \\ Universidad Regional Autónoma de los Andes, Quevedo \\ Ecuador \\ https://orcid.org/0000-0002-3060-736X
}

Recibido: 16 de mayo de 2020

Revisado: 12 de junio de 2020

Aprobado: 15 de julio de 2020

Publicado: 01 de agosto de 2020 
Revista Arbitrada Interdisciplinaria KOINONIA

Año 2020. Vol V. ํ⒉ Especial: Saber y Tecnología Popular Hecho el depósito de Ley: FA2016000010

ISSN: 2542-3088

FUNDACIÓN KOINONIA (F.K). Santa Ana de Coro. Venezuela.

José Enrique Guevara-Valle; Andrea Raquel Zúñiga-Paredes; Edmundo José Jalón-Arias

\title{
RESUMEN
}

La investigación tiene por objetivo diseñar un aplicativo web para la creación y toma de evaluaciones a estudiantes del nivel secundario en el Cantón de Quevedo del Ecuador. Se fundamentó desde el enfoque cuantitativo, con un tipo descriptiva. El $55 \%$ de los docentes encuestados consideran necesario aplicar software educativo con fines de evaluar los aprendizajes en razón del enfoque de las TIC, propiciándose así una educación integral y de calidad. El aplicativo web consiste en la toma de evaluaciones con el fin de mejorar la calidad en el servicio que presta la institución y llevarla a la excelencia académica. El proyecto fue desarrollado con lenguajes de programación como PHP, HTML, SQL, la página consta desde el inicio con una interfaz amigable, un login donde el maestro o estudiante podrá tener acceso a la página ingresando sus datos ya registrados.

Descriptores: Evaluación de conocimientos anteriores; evaluación del estudiante; rendimiento escolar; programa informático didáctico. (Palabras tomadas del Tesauro UNESCO).

\begin{abstract}
The objective of the research is to design a web application for the creation and taking of evaluations to high school students in the Canton of Quevedo of Ecuador. It was based on the quantitative approach, with a descriptive type. 55\% of the teachers surveyed consider it necessary to apply educational software in order to evaluate learning due to the ICT approach, thus promoting a comprehensive and quality education. The web application consists of taking evaluations in order to improve the quality of the service provided by the institution and lead it to academic excellence. The project was developed with programming languages such as PHP, HTML, SQL, the page has a friendly interface from the beginning, a login where the teacher or student can access the page by entering their already registered data.
\end{abstract}

Descriptors: Prior learning evaluation; student evaluation; academic achievement; educational software. (Words taken from the UNESCO Thesaurus). 


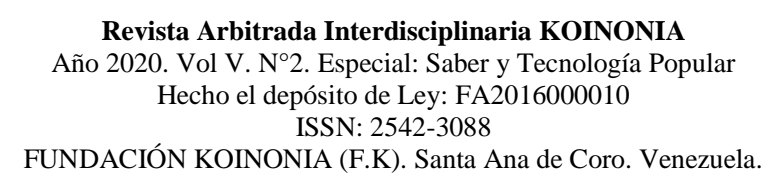

José Enrique Guevara-Valle; Andrea Raquel Zúñiga-Paredes; Edmundo José Jalón-Arias

\section{INTRODUCCIÓN}

La evaluación en la educación ha sido desde siempre una constante construcción de procesos que contribuyen al desarrollo de la enseñanza y el aprendizaje, enfocado en el fin de la educación que es la transformación del ser humano en positivo, se dirá entonces que la evaluación es el juicio que el docente emite sobre una tarea, examen u otro aspecto que amerite darle una estimación o valor. (Mendieta, et al., 2016). Por lo tanto, evaluación es la valoración que se lleva a cabo, a partir de la observación, análisis, de los datos, ya sea del proceso de aprendizaje con el fin de tomar decisiones orientadas a mejorar el trabajo y ayudar a progresar (evaluación para el aprendizaje y como aprendizaje), o de la consecución de unos objetivos o del nivel de dominio en el uso de la lengua, con el fin de llevar a cabo un juicio o una calificación (evaluación del aprendizaje). (Pérez-Pino, et al., 2017).

La educación en su evolución ha incorporado la tecnología (TIC) como una forma de mediar el conocimiento entre el discente y profesor, siendo recurrente implementar un enfoque pedagógico basado en el constructivismo social como esencia de la formación y evaluación de los aprendizajes, siendo necesario compaginar tanto temas como evaluación en función de las herramientas, estrategias didácticas en relación a las TIC (Hernández, et al., 2019).

Por lo tanto, podría existir similitud entre la evaluación de los aprendizajes del modelo tradicional educativo con las TIC, sin embargo, se requiere que esta última diseñe la ruta evaluativa en función de las características de la audiencia (estudiantes), así existirá mayor posibilidad de germinar un aprendizaje significativo en función de demostrar las capacidades aprendidas en el ambiente de aprendizaje (Quevedo-Álava, et al, 2020), siendo recurrente contar con aplicaciones o software pertinentes en razón de lo descrito anteriormente con lo que podrá desarrollarse una educación inclusiva, de calidad y en equidad de condiciones (Muguercia-Bles, et al., 2017).

En este sentido, se tiene que el modelo educativo en el Ecuador en palabras de (CedeñoTroya, 2017), no ha tenido un cambio sustancial en el ámbito tecnológico. En el proceso 


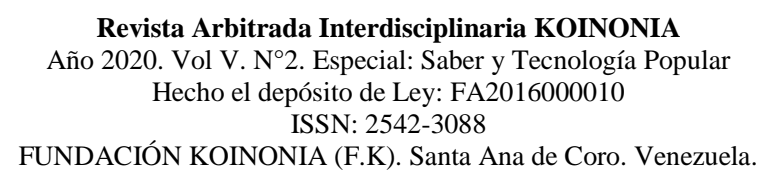

José Enrique Guevara-Valle; Andrea Raquel Zúñiga-Paredes; Edmundo José Jalón-Arias

de enseñanza - aprendizaje el esquema tradicional trae consigo un gran número de problemas como: falta de honestidad en los estudiantes, alto consumo de papel, demoras en la calificación y entrega de resultados (Arroyo-Carrera, et al., 2020), en este sentido existen muchas instituciones que presentan una indagación documentada sobre estas situaciones, pero el enfoque central de la presente investigación será el proceso de evaluación (Saltos-Cedeño, et al., 2020).

El gobierno actual persigue un cambio total en cada uno de los procesos que conforman el modelo educativo, orientándose a una educación con calidad al nivel de los países desarrollados (Clavijo-Castillo \& Bautista-Cerro, 2020), para lo cual es importante con el apoyo de software educativos que contribuyan a realizar evaluaciones en función de la esencia del enfoque basado en TIC, así como del aprendizaje de los estudiantes (GarcíaSánchez, et al., 2016). En razón de lo planteado, se procedió a realizar una investigación en las instituciones educativas de educación secundaria del cantón Quevedo del Ecuador, con la finalidad de conocer el proceso de evaluación de los aprendizajes desde la vertiente de las TIC.

La actual investigación tiene por objetivo diseñar un aplicativo web para la creación y toma de evaluaciones a estudiantes del nivel secundario en el Cantón de Quevedo del Ecuador.

\section{MÉTODO}

La investigación se fundamentó desde el enfoque cuantitativo, con un tipo descriptiva, diseño de campo no experimental, en este sentido, la muestra poblacional estuvo constituida por 62 docentes de educación secundaria de la Unidad Educativa Ciudad de Quevedo, a quienes se les aplicó encuesta y cuestionario en escala Likert vía online, el cual fue validado por el juicio de tres expertos, se realizó prueba piloto para el cálculo del coeficiente Alfa de Cronbach, dando como resultado 0,89 lo cual permitió catalogar como confiable el instrumento, mientras que los datos recopilados fueron procesados mediante estadística descriptiva (Hernández-Sampieri, et al., 2014). 


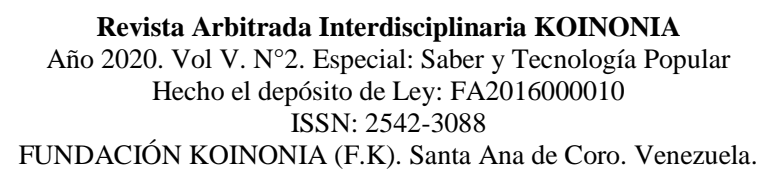

José Enrique Guevara-Valle; Andrea Raquel Zúñiga-Paredes; Edmundo José Jalón-Arias

\section{RESULTADOS}

El 55\% de los docentes encuestados consideran necesario aplicar software educativo con fines de evaluar los aprendizajes en razón del enfoque de las TIC, propiciándose así una educación integral y de calidad. Mientras que el $65 \%$ considera importante capacitarse para manejar efectivamente los programas educativos basados en la TIC, de ese modo, existe mayor probabilidad de promover una comunicación asertiva en el proceso educativo entre los estudiantes y docentes.

Por otro lado, el $80 \%$ considera que los programas educativos deben tener concordancia con las necesidades académicas de los estudiantes, siendo que estos deben ser diseñados compaginando los propósitos académicos con fines lúdicos, lo cual permitirá ser atractivo a la percepción cognitiva de los discentes.

\section{PROPUESTA}

En la presente investigación se diseñó un sistema web con el uso del software Dreamweaver que permite la construcción y diseño de sitios web; plantillas de CSS para los estilos de diseño; javascript para ejecutar animación y validar formularios y base de datos MySql para el almacenamiento de los datos. La investigación ayudó a recolectar la información necesaria y poder visualizar diferentes páginas web de evaluación, el desarrollo de la página web de creación y toma de evaluaciones a estudiantes se logró desarrollar con los datos de información de varios docentes de bachillerato de la Unidad Educativa Ciudad de Quevedo, la cual manifestaron que en ocasiones es difícil encontrar un software o página web que se acople a las necesidades que requieren, dado que el gobierno actual no maneja una política global ni plataforma para evaluación estudiantil. En base a lo expuesto se encontró un plus en el desarrollo del aplicativo, que constó con login que permite el ingreso del docente y a su vez el del estudiante como se muestra en la figura 1, el trabajo del docente donde se registran preguntas y forma de evaluar y dejando que la aplicación gestione el resto de procesos con calificación obtenida. De esta manera habrá un control de la fecha en la que realizó la evaluación y la calificación 
correspondiente. Teniendo en cuenta que este aplicativo se desarrolló en PHP seguidos de líneas HTML, sus etiquetas CSS que permiten crear las plantillas donde el aplicativo será intuitivo para los espectadores, y consta con responsive haciéndolo compatible para dispositivos móviles. El producto obtenido se expone a continuación:

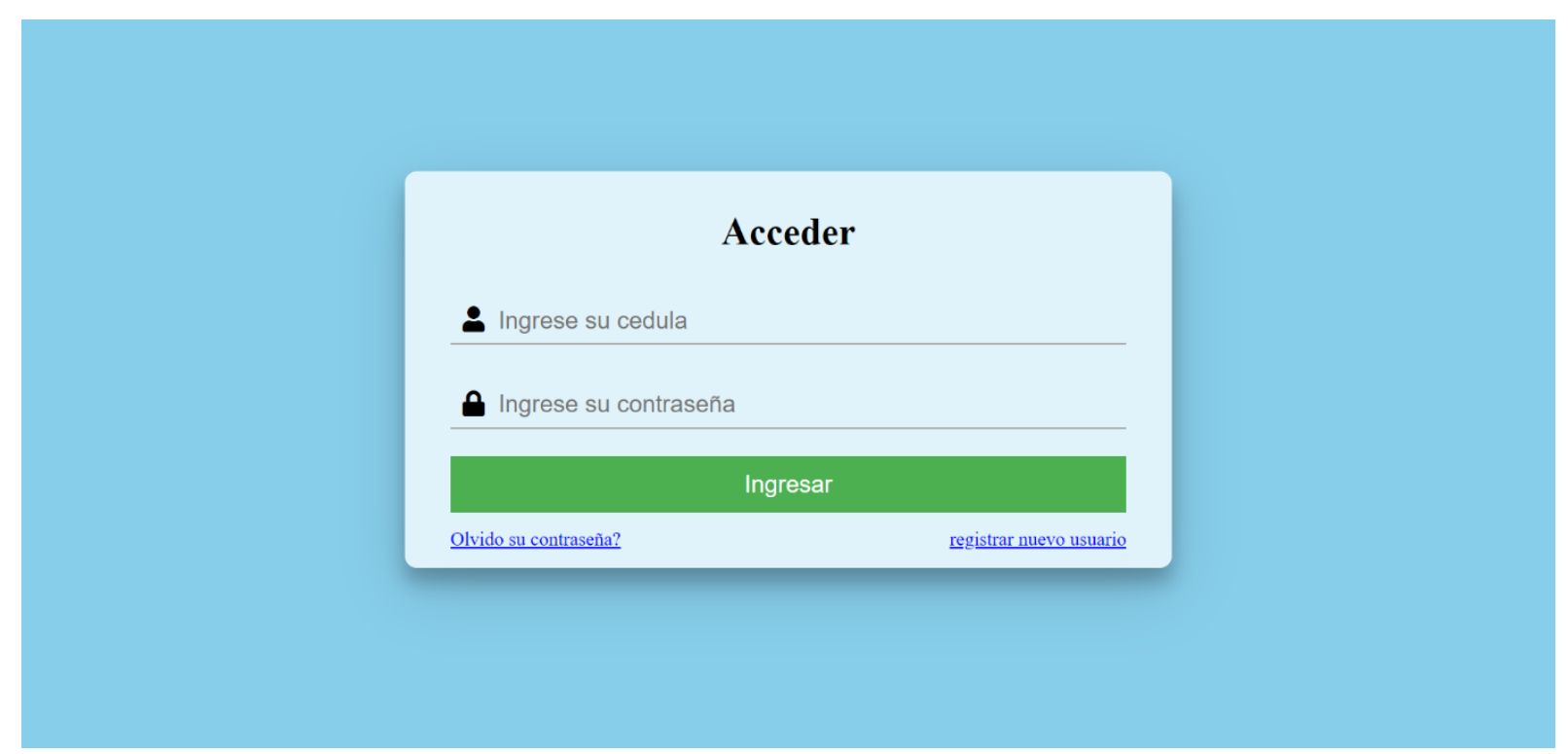

Figura 1. Login de Aplicativo Web.

Fuente: Los autores.

Como interfaz principal tenemos el login de acceso al aplicativo web con diferentes características donde podrá el maestro o estudiante realizar su registro e ingresar con su usuario o número de cedula y contraseña. 
Revista Arbitrada Interdisciplinaria KOINONIA

Año 2020. Vol V. N². Especial: Saber y Tecnología Popular Hecho el depósito de Ley: FA2016000010

ISSN: 2542-3088

FUNDACIÓN KOINONIA (F.K). Santa Ana de Coro. Venezuela.

José Enrique Guevara-Valle; Andrea Raquel Zúñiga-Paredes; Edmundo José Jalón-Arias

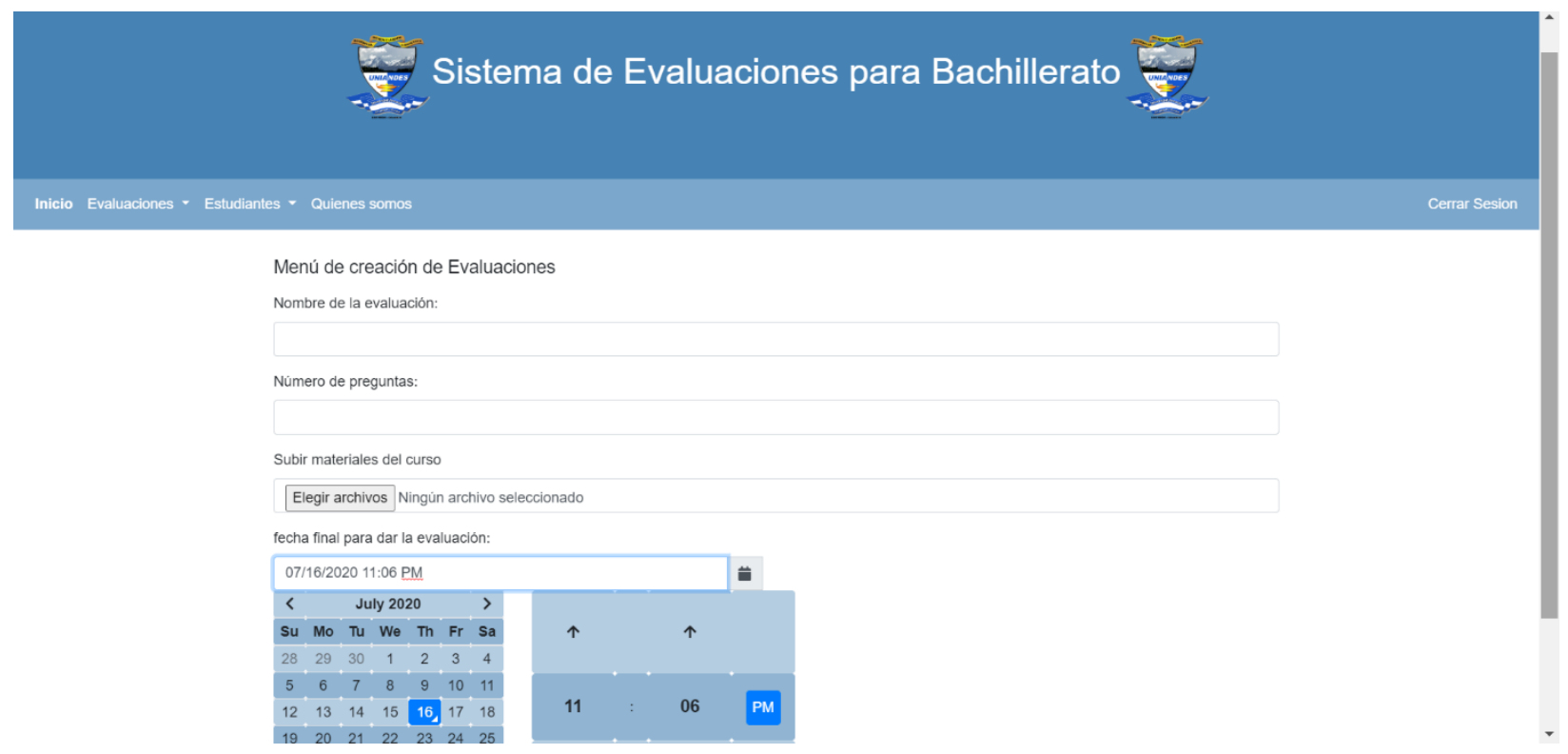

Figura 2. Menú de Creación de Evaluaciones.

Fuente: Los autores.

En esta sección el docente podrá crear las evaluaciones donde elegirá cuantas preguntas va a gestionar, posterior a esto también tendrá la opción de agregar material didáctico que servirá como retroalimentación para el estudiante. Para la elaboración de preguntas primero se estipulará un tema evaluativo, este constará con fecha y hora para poder realizarla. 


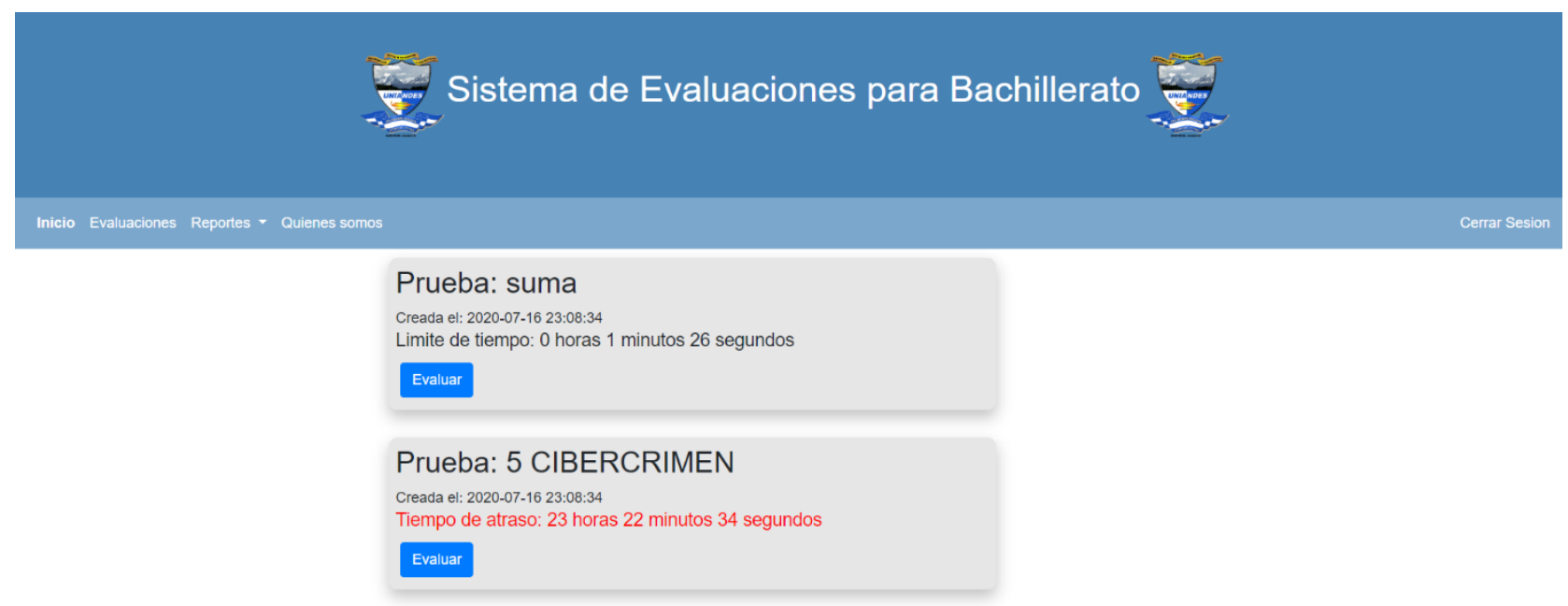

Figura 3. Evaluaciones Pendientes en Perfil Estudiantil. Fuente: Los autores.

El estudiante al ingresar a su cuenta personal podrá visualizar las evaluaciones pendientes a realizar. Una vez concluida la evaluación el usuario tendrá la opción de ver su calificación.

Se puede apreciar en la figura 1, un ingreso de usuario único que les permitirá la entrada al sistema haciéndole de fácil acceso, con una interfaz sencilla y amigable tanto para el docente como para el estudiante.

Posteriormente en la figura 2 se recalca la creación de evaluaciones donde el magistral incluso podrá agregar material de apoyo para el estudiante, el aplicativo web consta con fecha y hora en cada examen para así poder verificar su cumplimiento haciendo énfasis a una actitud humana como es la puntualidad.

Los datos estadísticos obtenidos en las encuestas realizadas fueron satisfactorios como se da a relucir en las ilustraciones cuatro y cinco por la buena aceptación que tuvo la página, posterior a la investigación consolidada, permitiendo a los autores una proyección para nuevas instituciones educativas. 


\section{Revista Arbitrada Interdisciplinaria KOINONIA \\ Año 2020. Vol V. №2. Especial: Saber y Tecnología Popular Hecho el depósito de Ley: FA2016000010 \\ ISSN: 2542-3088 \\ FUNDACIÓN KOINONIA (F.K). Santa Ana de Coro. Venezuela.}

José Enrique Guevara-Valle; Andrea Raquel Zúñiga-Paredes; Edmundo José Jalón-Arias

\section{CONCLUSIÓN}

Se evidenció deficiencias en el proceso automatizado de evaluaciones en algunas instituciones educativas, presentando poco dominio en el proceso de evaluación, llegando a cursos de actualización para el correcto manejo de la aplicación de la evaluación, considerando favorable en los logros de aprendizajes significativos en el proceso de enseñanza aprendizaje.

El problema latente que sufren la mayoría de instituciones educativas es el tiempo que toma poder realizar un examen de manera escrita, gastando recursos innecesarios y afectando el medio ambiente. La investigación contribuyó de gran manera a obtener una solución rápida y precisa por medio de la tecnología de información, usando el internet como medio de comunicación.

El aplicativo web consiste en la toma de evaluaciones con el fin de mejorar la calidad en el servicio que presta la institución y llevarla a la excelencia académica. El proyecto fue desarrollado con lenguajes de programación como PHP, HTML, SQL, la página consta desde el inicio con una interfaz amigable, un login donde el maestro o estudiante podrá tener acceso a la página ingresando sus datos ya registrados, si el estudiante no se encuentra registrado deberá ir a la sección de registro y cumplir con el proceso de registro de datos correctamente, el docente será el encargado de aceptar las notificaciones de estudiante nuevo. Una vez aceptado el alumno ya puede ingresar a su cuenta y así realizar las evaluaciones correspondientes; el sistema además cuenta visualización de calificaciones, gestión de evaluaciones con el perfil docente y calificación automática.

\section{FINANCIAMIENTO}

No monetario

\section{AGRADECIMIENTO}

A la Universidad Regional Autónoma de los Andes, Quevedo; por apoyar la investigación. 
Revista Arbitrada Interdisciplinaria KOINONIA

Año 2020. Vol V. ํ⒉ Especial: Saber y Tecnología Popular Hecho el depósito de Ley: FA2016000010

ISSN: 2542-3088

FUNDACIÓN KOINONIA (F.K). Santa Ana de Coro. Venezuela.

José Enrique Guevara-Valle; Andrea Raquel Zúñiga-Paredes; Edmundo José Jalón-Arias

\section{REFERENCIAS CONSULTADAS}

Arroyo-Carrera, E., Loor-Santos, M., Mendoza-Mera, J., \& Solorzano-Zambrano, M. (2020). Gestión de aprendizaje creativo mediante la Herramienta Powtoon en estudiantes de lengua y literatura [Creative learning management through the Powtoon Tool in language arts students]. EPISTEME KOINONIA, 3(5), 253-269. http://dx.doi.org/10.35381/e.k.v3i5.775

Cedeño-Troya, F. (2017). Diseño de un Software de evaluaciones en línea para los estudiantes y docentes de grado en la Universidad Tecnológica Empresarial de Guayaquil [Design of an online assessment software for undergraduate students and teachers at the Universidad Tecnológica Empresarial de Guayaquil]. Espirales Revista Multidisciplinaria de investigación, 1(9), 1-14.

Clavijo-Castillo, R, \& Bautista-Cerro, M. (2020). La educación inclusiva. Análisis y reflexiones en la educación superior ecuatoriana [Inclusive education. Analysis and reflections in Ecuadorian Higher Education]. ALTERIDAD. Revista de Educación, 15(1), 113-124. https://dx.doi.org/10.17163/alt.v15n1.2020.09

García-Sánchez, E, Vite-Chávez, O, Navarrate-Sánchez, M, García-Sánchez, M, \& Torres-Cosío, V. (2016). Metodología para el desarrollo de software multimedia educativo MEDESME. CPU-e. Revista de Investigación Educativa, (23), 216-226.

Hernández, R, Sánchez-Cáceres, I, Zarate-Hermoza, J, Medina-Coronado, D, Loli-Poma, T, \& Arévalo-Gómez, G. (2019). Tecnología de Información y Comunicación (TIC) y su práctica en la evaluación educativa [Information and Communication Technology (ICT) and its Practice in Educational Evaluation]. Propósitos y Representaciones, 7(2), 1-5. https://dx.doi.org/10.20511/pyr2019.v7n2.328

Hernández-Sampieri, R., Fernández-Collado, C., Baptista-Lucio, P. (2014). Metodología de la Investigación. [Investigation methodology]. México: Editorial Mc - Graw Hill Interamericana. Sexta edición.

Mendieta, L., Chamba, J., \& Mieles, M. (2016). Estrategias pedagógicas para la evaluación del estudiante en el nivel superior [Pedagogical strategies for educational assessment of student in higher level]. Ciencia y Desarrollo, 19 (2): 33-42.

Muguercia-Bles, A, \& Negret, Y, \& Benito-Valenciano, V, \& De-la-Torre-Vega, G. (2017). Software educativo didáctico para el aprendizaje de la asignatura Informática [Didactic educational software for the learning of the Computer science subject]. MEDISAN, 21(8), 1094-2001. 
Revista Arbitrada Interdisciplinaria KOINONIA

Año 2020. Vol V. N². Especial: Saber y Tecnología Popular Hecho el depósito de Ley: FA2016000010

ISSN: 2542-3088

FUNDACIÓN KOINONIA (F.K). Santa Ana de Coro. Venezuela.

José Enrique Guevara-Valle; Andrea Raquel Zúñiga-Paredes; Edmundo José Jalón-Arias

Pérez-Pino, M, Enrique-Clavero, J, Carbó-Ayala, J, \& González-Falcón, M. (2017). La evaluación formativa en el proceso enseñanza aprendizaje [Formative evaluation in the teaching-learning process]. EDUMECENTRO, 9(3), 263-283.

Quevedo-Álava, R., Corrales-Moreno, L., Palma-Delgado, G., \& Mendoza-Suárez, G. (2020). Psicopedagogía y TIC en período de COVID-19. Una reflexión para el aprendizaje significativo [Psychopedagogy and ICT in the COVID-19 period. A reflection for meaningful learning]. EPISTEME KOINONIA, 3(5), 202-222. http://dx.doi.org/10.35381/e.k.v3i5.769

Saltos-Cedeño, A., Vallejo-Valdivieso, P., \& Moya-Martínez, M. (2020). Innovación en educación matemática de básica superior durante el confinamiento por COVID19 [Innovation in higher basic mathematics education during confinement by COVID-19]. $\quad$ EPISTEME KOINONIA, $3(5), \quad$ 142-161. http://dx.doi.org/10.35381/e.k.v3i5.723 